\title{
REAL AND COMPLEX CHEBYSHEV APPROXIMATION ON THE UNIT DISK AND INTERVAL
}

\author{
BY MARTIN H. GUTKNECHT AND LLOYD N. TREFETHEN ${ }^{1}$
}

We announce the resolution of a number of outstanding questions regarding real and complex Chebyshev (supremum norm) approximation by rational functions on a disk and on an interval. The proofs consist mainly of symmetry arguments applied to explicit examples. The most important results: complex rational best approximations on a disk are in general not unique; real functions on an interval can in general be approximated arbitrarily much better by complex rational functions than by real ones. Details will appear in $[3,8]$.

1. Notation. Define $\Delta=\{z:|z| \leq 1\}, A_{\Delta}=\{f$ : continuous on $\Delta$, analytic in the interior $\},\|f\|_{\Delta}=\sup \{|f(z)|: z \in \Delta\}$. Let $m \geq 0, n \geq 1$ be integers (all questions considered below become trivial for $n=0$ ), and let $R_{m n}$ be the space of complex rational functions of type $(m, n)$. Define $A_{\Delta}^{r}=\left\{f \in A_{\Delta}: f(\bar{z})=\right.$ $\overline{f(z)}\}, R_{m n}^{r}=\left\{r \in R_{m n}: r(\bar{z})=\overline{r(z)}\right\}$, and for $f \in A_{\Delta}$,

$$
E_{m n}(f ; \Delta)=\inf _{r \in R_{m n}}\|f-r\|_{\Delta}, \quad E_{m n}^{r}(f ; \Delta)=\inf _{r \in R_{m n}^{r}}\|f-r\|_{\Delta} .
$$

It is known that these infima are attained (proof by a normal families argument due to Walsh [10]), and we let $N_{m n}(f ; \Delta)$ and $N_{m n}^{r}(f ; \Delta)$ denote the number (finite or infinite) of best approximations ( $B A$ 's) to $f$.

Finally, set $I=[-1,1]$, and let $A_{I}, A_{I}^{r},\|\cdot\|_{I}, E_{m n}(f ; I), E_{m n}^{r}(f ; I)$, $N_{m n}(f ; I), N_{m n}^{r}(f ; I)$ be defined analogously. $\left(A_{I}\right.$ and $A_{I}^{r}$ are just the sets of continuous complex and real functions on $I$, respectively.)

2. Nonuniqueness. It is a classical result due to Achieser that $N_{m n}^{r}(f ; I)=$ 1 for all $m, n$ and all $f \in A_{I}^{r}$. But Lungu [4] (on proposal of A. A. Gončar) and independently Saff and Varga [6, 7] found that for all $m$ and $n$ there exists $f \in$ $A_{I}^{r}$ with $E_{m n}(f ; I)<E_{m n}^{r}(f ; I)$, so that by symmetry necessarily $N_{m n}(f ; I) \geq$ 2. Ruttan [5] even gave an example with $N_{11}(f ; I)=\infty$. However, the analogous questions for the disk have been open $[\mathbf{2}, \mathbf{9}]$. We claim [3]:

THEOREM 1. $\forall m, n, \forall K \geq 1, \exists f \in A_{\Delta}$ such that $N_{m n}(f ; \Delta) \geq K$.

THEOREM 2. $\forall m, n$ with $m=0$ or $n=1, \exists f \in A_{\Delta}^{r}$ such that $E_{m n}(f ; \Delta)$ $<E_{m n}^{r}(f ; \Delta)$.

THEOREM 3. $\forall m, n, \exists f \in A_{\Delta}^{r}$ such that $N_{m n}^{r}(f ; \Delta)>1$.

Received by the editors October 1, 1982.

1980 Mathematics Subject Classification. Primary 30E10; Secondary 41A20.

${ }^{1}$ Supported by a National Science Foundation Mathematical Sciences Postdoctoral Fellowship. 
(We believe that the assertion of Theorem 2 probably holds for arbitrary $m$ and $n$.) For $(m, n)=(0,1)$ and $K=2$, these claims can be established as follows. The function $f(z)=z+z^{3}$ attains maximum modulus at the points \pm 1 , with $f(1)=-f(-1)=2$. An approximant from $R_{01}^{r}$ with no pole on $\Delta$ must have the same sign at -1 as at +1 , which implies that 0 is a BA in $R_{01}^{r}$, hence $E_{01}^{r}(f ; \Delta)=2$. On the other hand $r(z)=1 /(z-2 i) \in R_{01}$ has $\operatorname{Re} r(1)>0, \operatorname{Re} r(-1)<0$, so for small enough $\delta,\|f-\delta r\|_{\Delta}<\|f\|_{\Delta}$, hence $E_{01}(f ; \Delta)<E_{01}^{r}(f ; \Delta)$ (Theorem 2 ); hence by symmetry $N_{01}(f ; \Delta) \geq 2$ (Theorem 1). Similarly with $f(z)=z-z^{3}$ one shows that any BA from $R_{01}^{r}$ necessarily has a finite pole at $z_{0}$ with either $z_{0}>0$ or $z_{0}<0$, and then symmetry implies that there is another BA with a pole at $-z_{0}$ (Theorem 3 ).

3. Padé approximation; small disks and intervals. Let $J$ be the interval $[0,1]$. For $f$ analytic in a neighborhood of the origin, and for fixed $m, n$ and any sufficiently small $\epsilon>0$, let $r_{\epsilon \Delta}^{*}, r_{\epsilon I}^{*}$, and $r_{\epsilon J}^{*}$ denote BA's to $f$ in $R_{m n}$ on $\epsilon \Delta, \epsilon I$, and $\epsilon J$, respectively. Let $r^{p}$ be the Padé approximant to $f$ of type $(m, n)$, whose coefficients have a connection to the $n \times n$ Hankel matrix $H=\left(\alpha_{m-n+i+j-1}\right)_{i, j=1}^{n}$, where $f(z)=\alpha_{0}+\alpha_{1} z+\alpha_{2} z^{2}+\cdots\left(\alpha_{k}=0\right.$ for $\left.k<0\right)$. Walsh showed in 1964 and 1974:

THEOREM [11, 12]. If $\operatorname{det} H \neq 0$, then $r_{\epsilon J}^{*} \rightarrow r^{p}$ and $r_{\epsilon \Delta}^{*} \rightarrow r^{p}$ as $\epsilon \rightarrow 0$.

By $r^{*} \rightarrow r^{p}$ we mean that the functions $r^{*}$ approach $r^{p}$ uniformly on compact sets containing no poles of $r^{p}$.

Walsh did not determine whether the condition $\operatorname{det} H \neq 0$ is necessary, and Chui et al. [1] have shown that if attention is restricted to approximation in $R_{m n}^{r}$ of a real function on $J$, it is not. But we claim [3]

THEOREM 4. $\forall m, n, \exists f \in A_{\Delta}$ for which $r_{\epsilon \Delta}^{*} \not f r^{p}$ as $\epsilon \rightarrow 0$.

TheOREM 5. $\exists m, n, f \in A_{I}^{r}$ for which $r_{\epsilon I}^{*} \not \subset r^{p}$ as $\epsilon \rightarrow 0$.

These theorems are proved by picking $f$ as in the nonuniqueness proofs such that $r^{p}=0$, but such that $r_{\epsilon}^{*}$ has a pole. One then shows that as $\epsilon \rightarrow 0$ this pole approaches the origin, which implies $r_{\epsilon}^{*} \not r^{p}$.

4. Degree of approximation. Since $E<E^{r}$ can occur on both $I$ and $\Delta$, it is natural to ask whether the ratios

$$
\gamma_{m n}^{I}=\inf _{f \in A_{I}^{r} \backslash R_{m n}^{r}} \frac{E_{m n}(f ; I)}{E_{m n}^{r}(f ; I)}, \quad \gamma_{m n}^{\Delta}=\inf _{f \in A_{\Delta}^{r} \backslash R_{m n}^{r}} \frac{E_{m n}(f ; \Delta)}{E_{m n}^{r}(f ; \Delta)}
$$

are zero or positive, and if positive, how small. Such a question was raised by Saff and Varga for the interval $I[6,7,8]$ and considered further by Bennet, Rudnick, and Vaaler, and by Ruttan [5] in the case $m=n=1$ and by Ellacott [2] in the case $m \geq n$. No examples have been found heretofore with $E / E^{r}<$ $1 / 2$, but we claim $[8]$

THEOREM 6. $\gamma_{m n}^{I}=0$ for $n \geq m+3$.

THEOREM 7. $\gamma_{0 n}^{\Delta}=0$ for $n \geq 4$. 
The idea behind the proofs is that one or more complex poles near the domain of approximation can introduce an approximate sign change, thereby simulating the behavior of a real zero. Thus for Theorem 6 with $m=0$, consider

$$
\phi(x)=\frac{2 \epsilon}{[x+(1+\epsilon)][x-(1+\epsilon)][x-i \sqrt{\epsilon}]} \in R_{03}
$$

and $f(x)=\operatorname{Re} \phi(x)$. Then $\|f\|_{I}=f(-1)=-f(1)=1+O(\epsilon)$, so the equioscillation theorem implies that 0 is the $\mathrm{BA}$ in $R_{0 n}^{r}$, with $E_{0 n}^{r}(f ; I)=1+O(\epsilon)$, while on the other hand $E_{0 n}(f ; I) \leq\|f-\phi\|_{I}=\|\operatorname{Im} \phi\|_{I}=O(\sqrt{\epsilon})$. Taking $\epsilon \rightarrow 0$ gives $\gamma_{0 n}^{I}=0$.

However $\gamma_{m n}^{I}=0$ cannot hold for all $(m, n)$, for we have also shown [8]:

THEOREM 8. $\gamma_{01}^{I}>0$.

We suspect that the result of Theorem 6 is sharp.

CONJECTURE. $\gamma_{m n}^{I}=0$ if and only if $n \geq m+3$.

5. General regions. The same ideas can be applied to obtain various results for approximation on more general regions in $\mathbf{C}$. For example, let $\Omega$ be a Jordan region with $\Omega=\bar{\Omega}$ whose boundary $\partial \Omega$ is differentiable at its two points of intersection with $\mathbf{R}$, hence forms a right angle to $\mathbf{R}$ at these points. Then Theorem 7 (hence also Theorems 1, 2) extends as follows [8]:

THEOREM 9. $\gamma_{0 n}^{\Omega}=0$ for $n \geq 4$.

On the other hand Theorem 8 can also be generalized.

THEOREM 10. $\gamma_{01}^{\Omega}>0$; in particular, $\gamma_{01}^{\Delta}>0$.

NOTE ADDED IN PROOF. Several additional results have been obtained concerning the Padé and best approximation questions discussed in $\S 3$. In particular, further explicit examples show that $r_{\epsilon J}^{*} \not \subset r^{p}$ and $r_{\epsilon I}^{*} \nrightarrow r^{p}$ can occur even for real approximation of real functions; thus the result of [1] quoted above is false. These matters will be discussed in a future publication.

\section{REFERENCES}

1. C. K. Chui, O. Shisha and P. W. Smith, Padé approximants as limits of best rational approximants, J. Approx. Theory 12 (1974), 201-204.

2. S. W. Ellacott, A note on a problem of Saff and Varga concerning the degree of complex approximation to real valued functions, Bull. Amer. Math. Soc. (N.S.) 6 (1982), 218-220.

3. M. H. Gutknecht and L. N. Trefethen, Nonuniqueness of rational Chebyshev approximations on the unit disk, J. Approx. Theory (to appear).

4. K. N. Lungu, Best approximation by rational functions, Mat. Z. 10 (1971), 11-15. (Russian)

5. A. Ruttan, On the cardinality of a set of best complex rational approximations to real function, Padé and Rational Approximation (E. B. Saff and R. S. Varga, eds.), Academic Press, New York, 1977, pp. 303-319.

6. E. B. Saff and R. S. Varga, Nonuniqueness of best approximating complex rational.functions, Bull. Amer. Math. Soc. 83 (1977), 375-377.

7. __ Nonuniqueness of best complex rational approximations to real functions on real intervals, J. Approx. Theory 23 (1978), 78-85. 
8. L. N. Trefethen and M. H. Gutknecht, Real vs. complex rational Chebyshev approximation on an interval, Trans. Amer. Math. Soc. (submitted).

9. R. S. Varga, Topics in polynomial and rational interpolation and approximation, Les Presses de l'Université de Montréal, Montréal, 1982.

10. J. L. Walsh, Interpolation and approximation by rational functions in the complex domain, 5th. ed., Amer. Math. Soc. Colloq. Publ., vol. 20, Amer. Math. Soc., Providence, R.I., 1966.

11. __ Padé approximants as limits of rational functions of best approximation, J. Math. Mech. 13 (1964), 305-312.

12. _ Padé approximants as limits of rational functions of best approximation, real domain, J. Approx. Theory 11 (1974), 225-230.

SEMINAR Für ANGEWANDTE MATHEMATIK, ETH ZURICH, SWITZERLAND

Courant INSTITUTE of MATHEMATICAL SCIENCES, NEW YORK UNIVERSITY, NEW YORK, NEW YORK 10012 